\title{
Um novo modelo dinâmico para o tráfego em regiões: proposta conceitual e estudo de caso com dados reais de Belo Horizonte
}

\author{
Ramon da Cunha Lopes* Marcio Fantini Miranda** \\ Paulo Eduardo Maciel de Almeida* \\ ${ }^{*}$ Lab. de Sist. Inteligentes, CEFET-MG, Av. Amazonas, 7675-CEP: \\ 30.510-000, Belo Horizonte, Minas Gerais, Brasil (e-mail: \\ $\{$ ramon,pema\}@decom.cefetmg.br) \\ ** COLTEC / UFMG, Av. Antônio Carlos, 6627 - CEP: 31270-901, \\ Belo Horizonte, Minas Gerais, Brasil (e-mail: fantini@ufmg.br)
}

\begin{abstract}
This paper presents an approach for dynamic modeling of a region composed of several adjacent traffic intersections and proposes a performance metric derived from classical energy concepts to evaluate traffic systems by means of a control system that adjusts green times of traffic lights. To validate the proposal, the SUMO simulator is used. The results obtained suggest that the model in question can accurately capture the dynamic characteristics of the region under study and that the proposed metric has a good potential to be used as a tool for analysis and evaluation of traffic quality in an urban area.

Resumo: Este artigo apresenta uma abordagem para modelagem dinâmica de uma região composta por várias interseções de tráfego adjacentes e propõe uma métrica de desempenho derivada a partir de conceitos clássicos de energia para avaliar sistemas de tráfego em função da atuação de um sistema de controle nos tempos de verde dos semáforos. Para validação da proposta, é empregado o simulador SUMO. Os resultados obtidos sugerem que a modelagem em questão consegue capturar com precisão as características dinâmicas da região em estudo, e que a métrica proposta tem um bom potencial para ser usada como ferramenta de análise e avaliação da qualidade do tráfego em uma área urbana.
\end{abstract}

Keywords: Intelligent traffic system, Traffic Engineering, Dynamic modeling, Scalability

Palavras-chaves: Sistemas Inteligentes de Transporte, Engenharia de Tráfego, Modelagem Dinâmica, Escalabilidade.

\section{INTRODUÇÃO}

Nesse trabalho propõe-se uma nova abordagem para modelagem de sistemas de tráfego urbano. A proposta de modelagem relaciona conceitos da Engenharia de Tráfego com as teorias de controle e modelagem de sistemas dinâmicos e possui a importante característica de ser escalável, isto é, possibilita definir modelos matemáticos que representam desde uma interseção simples até grandes complexos de vias e cruzamentos. $\mathrm{O}$ modelo proposto, devido às suas características estruturais e numéricas, possibilita a utilização de técnicas modernas de análise e projeto de sistemas de controle para otimização do tráfego. Esta abordagem inclui o conceito de energia aplicado ao ambiente do tráfego urbano, que será usado como métrica para análise de desempenho e projeto de sistemas de controle baseados em otimização ou em técnicas usuais de controle discreto, tais como controladores preditivos, ou ainda no contexto de sistemas inteligentes de transporte.

O modelo proposto é baseado na associação de dois indicadores clássicos da engenharia de tráfego: a ocupação das vias e a velocidade média dos veículos, como discutido por

\footnotetext{
^ Os autores agradecem ao CefetMG pelo apoio financeiro.
}

Borg and Scerri (2014). Essas duas grandezas são relacionadas com o conceito de "energia da via". Neste sentido, parte-se da hipótese de que o equilíbrio da energia em uma determinada região urbana está associado à otimização da capacidade da infraestrutura de tráfego nesta região, visto que trata-se de um único modelo adaptativo que adequa os parâmetros em função da condição das vias entre fluxo sem retenção, congestionado ou saturado, enquanto os modelos clássicos tratam estas condições separadamente associando muitos parâmetros a serem ajustados Lopes et al. (2017).

Historicamente faz-se uma analogia do sistema de tráfego com o fluxo de fluidos em tubulações, como em Daganzo (1997). Neste trabalho, uma analogia similar é proposta, utilizando redes elétricas baseadas em capacitores e o estudo da energia associada (veja Mayer (2000)) para a formulação de um modelo que considera a ocupação das vias e a velocidade média dos veículos, associandoos com um análogo da energia elétrica. Neste sentido, surge a hipótese de que o equilíbrio da energia em uma determinada região urbana levaria a uma maximização da capacidade de fluxo desta região.

O artigo é organizado em 8 seções. Inicialmente apresentase o modelo básico de tráfego urbano. Na seção 2 o modelo 
é apresentado, com descrição da abordagem proposta. $\mathrm{Na}$ seção 3 são apresentados os conceitos de energia e densidade de energia em sistemas urbanos. Na seção 4 é apresentado o estudo de caso. São feitas comparações entre as relações da energia, a ocupação das vias e a velocidade média medida, e os resultados são analisados à luz dos conceitos de engenharia de tráfego. Após esta análise dos resultados (seção 5), na seção 6 algumas conclusões são apresentadas.

\section{UM NOVO MODELO PARA SISTEMAS DE TRÁFEGO URBANO}

O modelo usado neste trabalho baseia-se em resultados recentes, dentre os quais destacamos: $(i)$ Pecherkova et al. (2008), que apresentaram um modelo dinâmico de uma microregião usando uma descrição por espaço de estados e fizeram a validação do modelo usando o simulador AIMSUN; (ii)Homolova and Nagy (2005) que introduziram um modelo não linear que representa o tamanho da fila, a intensidade de veículos na entrada da via e ocupação medida nos detectores; (iii) Borg and Scerri (2014) que utilizaram um controle semafórico adaptativo baseado em programação dinâmica; e (iv) Borg and Scerri (2015) que apresentaram uma abordagem de controle usando uma estrutura hierárquica que tratava a detecção de filas de veículos em um trecho na proximidade de um cruzamento, quanto este trecho atingia a capacidade máxima. O modelo proposto é baseado na associação de dois indicadores clássicos da engenharia de tráfego: a ocupação das vias e a velocidade média dos veículos, como discutido por Borg and Scerri (2014).

\subsection{Modelo geral}

Seguindo os trabalhos acima mencionados, o modelo proposto é baseado na representação de sistemas no espaço de estados. Cada cruzamento de $n$ vias é representado por um modelo dinâmico no espaço de estados, discreto no tempo, na forma:

$$
\begin{gathered}
\dot{x}(k)=A x(k)+B(\zeta, \gamma, \mu) u(k)+E \eta(k), \\
x(k+1)=A x(k)+B(\zeta, \gamma, \mu) u(k)+E \eta(k),
\end{gathered}
$$

sendo $k$ o instante de amostragem, $x$ o vetor de estados de dimensão $n \times 1, u$ a entrada de controle, $\eta$ a entrada de perturbação, e $A, B, E$ matrizes de dimensões compatíveis, representando o sistema. Repare que a matriz $B($.$) é$ função do estados, tornando o sistema não linear.

O vetor de estados $x(k)=\left[\begin{array}{lll}\zeta_{k} & \gamma_{I k} & \phi\end{array}\right]^{T}$ é dado por três estados assim descritos:

- $\zeta_{k}$ representa o tamanho da fila: número de carros que aguardam em cada via para atravessar uma interseção no começo de cada ciclo em unidades de veículos [uv];

- $\gamma_{I k}$ é a intensidade de entrada: taxa de veículos que entram em cada via por ciclo [uv/c];

- $\phi$ representa a Ocupação: porção de tempo que o detector (laço magnético colocado na entrada e saída de cada segmento de via) está ocupado por um veículo $[\%]$.

\subsection{Modelagem de um cruzamento}

É proposto um modelo para um cruzamento de quatro vias, com fluxo em ambas direções. Consideram-se os estados relacionados com fluxo de veículos em todas as vias do cruzamento, como mencionado acima, e os tempos de verde de cada semáforo são as variáveis de atuação no sistema.

Para a descrição da dinâmica do tamanho da fila, $\zeta_{k}$, com dependência não-linear do fluxo, utilizamos:

$$
\zeta_{k+1}=\zeta_{k}+\gamma_{I k}-\gamma_{S k}\left(\zeta_{k}, \gamma_{I k}, \mu_{k}\right) \mu_{k}+w_{1 k}
$$

sendo a função não-linear:

$$
\gamma_{S k}\left(\zeta_{k}, \gamma_{I k}, \mu_{k}\right)=S-S e^{-\frac{\zeta_{k}+\gamma_{I k}}{S \mu_{k}}}
$$

com $S$ sendo a saturação de fluxo determinada pelas limitações da interseção, $\mu_{k}$ a razão de sinal verde por ciclo em cada semáforo e $W_{1 k}$ um ruído Gaussiano branco com média zero.

A intensidade média é modelada como um processo markoviano com média e desvio padrão conhecidos, dado por:

$$
\gamma_{I k+1}=\gamma_{I k}+w_{2 k}
$$

e a ocupação em cada período do ciclo é definida como um processo aleatório como relação linear entre a ocupação anterior, o tamanho anterior da fila e um dado desvio padrão:

$$
\phi_{k+1}=\kappa \zeta_{k}+\beta \phi_{k}+w_{3}
$$

A representação no espaço de estados é dada por:

$$
\begin{aligned}
{\left[\begin{array}{c}
\zeta_{k+1} \\
\gamma_{I k+1} \\
\phi_{k+1}
\end{array}\right]=} & \overbrace{\left[\begin{array}{lll}
1 & 1 & 0 \\
0 & 1 & 0 \\
\kappa & 0 & \beta
\end{array}\right]}^{A}\left[\begin{array}{c}
\zeta_{k} \\
\gamma_{I k} \\
\phi_{k}
\end{array}\right]+\overbrace{\left[\begin{array}{c}
-\gamma_{S k} \\
0 \\
0
\end{array}\right]}^{B} \mu_{k}+\left[\begin{array}{c}
w_{1 k} \\
w_{2 k} \\
w_{3 k}
\end{array}\right], \\
& {\left[\begin{array}{c}
\gamma_{I k+1} \\
\phi_{k+1}
\end{array}\right]=\overbrace{\left[\begin{array}{lll}
0 & 1 & 0 \\
0 & 0 & 1
\end{array}\right]}^{C}\left[\begin{array}{c}
\zeta_{k} \\
\gamma_{I k} \\
\phi_{k}
\end{array}\right]+\nu_{k}, }
\end{aligned}
$$

onde $\nu_{k}$ representa um sinal gaussiano de média zero.

Inicialmente, consideremos um cruzamento simétrico com entradas e saídas para todas as direções, com uma representação completa dos oito movimentos de entrada e saída, cujo diagrama é dado na Figura 1. Cada bloco implementa um movimento na via, e é representado por um modelo no espaço de estados. 


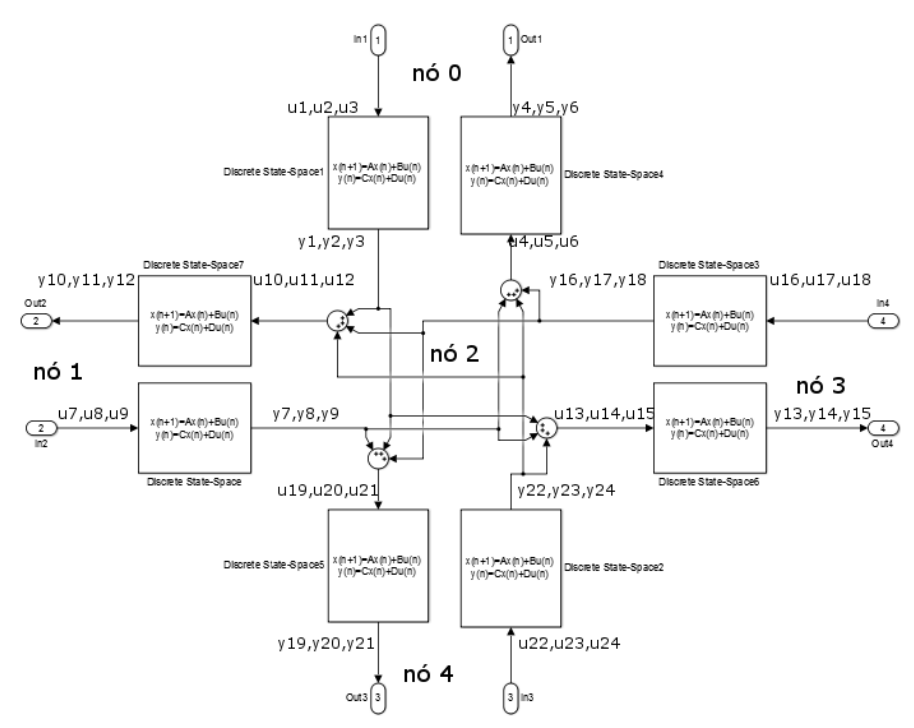

Figura 1. Associação de blocos no espaço de estados para representar um cruzamento de vias urbanas

\subsection{Metodologia de modelagem}

O processo para a obtenção do modelo escalável aqui proposto se baseia no conceito de energia da via, e é dividido em 3 etapas:

(1) Obter os parâmetros do segmento de via para modelos de primeira ordem (ver Lopes et al. (2017));

(2) Acoplar os segmentos de cada cruzamento;

(3) Conectar os cruzamentos para avaliação da distribuição de energia em uma região de tráfego.

Este processo será validado, a partir da Seção 4, por um estudo de caso que empregará dados reais de vias urbanas medidos em Belo Horizonte, MG, conforme descrito a seguir.

\section{MEDIDAS DE ENERGIA NO TRÁFEGO}

A energia de um cruzamento pode ser pensada como uma grandeza relacionada com a intensidade de veículos nas vias. Neste contexto, ela pode ser usada como um parâmetro relevante para medir e otimizar o fluxo numa determinada direção.

\subsection{Medida de desempenho baseada no cálculo da energia}

A analogia do fluxo de veículos com o fluxo em tubulações é uma metáfora largamente utilizada na literatura (p.ex. David et al. (2012), Halaoui (2010), Daganzo (1997) e Pedersen (2011)). Um aprofundamento desta analogia explora as equações que descrevem a dinâmica de fluidos e as leis da termodinâmica para expressar a energia medida em um sistema de tráfego (veja Nakrachi et al. (2012) e Liebe et al. (2011)). Esta função de energia total, representada por uma hamiltoniana (Morrison (1998)), é dada pela Equação 7:

$$
e_{t}=e_{k}+\epsilon(\rho)=\frac{1}{2} \rho v^{2}+\frac{c^{2}}{2 \rho_{c}}\left(\rho-\rho_{c}\right)^{2}
$$

sendo $e_{k}$ a densidade de energia cinética; $\epsilon(\rho)$ a densidade de energia interna; $c$ uma constante equivalente à velocidade do som, em média; $\rho_{c}$ a densidade crítica.

$\epsilon(\rho)$ deve se anular com o regime estacionário, conforme discutido por David et al. (2012). Esta função tem uma forma normalizada, como mostrado na Figura 2:

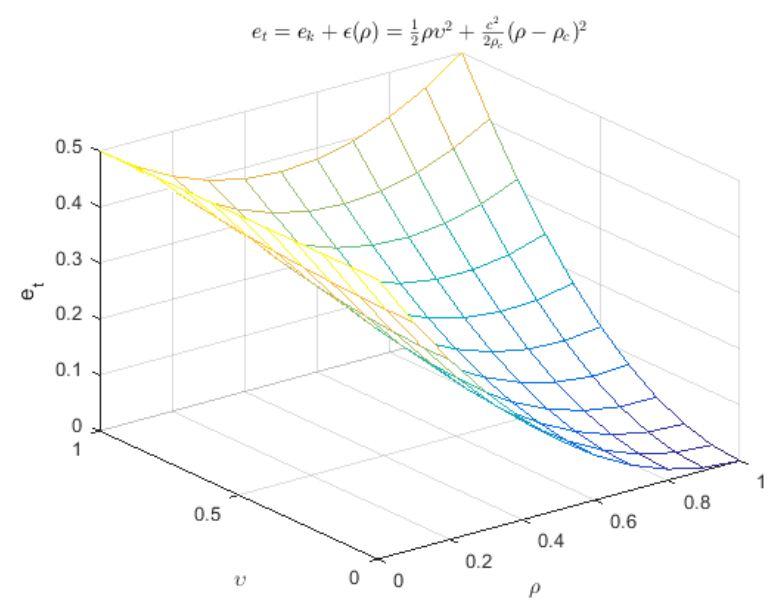

Figura 2. Função de energia normalizada.

Considerando os estados definidos na subseção 2.2 com as equações (1) e (2), temos a equação de energia em função dos estados $\phi$ e $\gamma$ dada por:

$$
e_{t}=e_{k}+\epsilon(\phi)=\frac{1}{2} \phi \gamma^{2}+\frac{c^{2}}{2 \phi_{c}}\left(\phi-\phi_{c}\right)^{2},
$$

que foi obtida trocando-se, na equação $7, \rho$ e $v$ por, respectivamente, $\phi$ e $\gamma$, sendo $\phi_{c}$ a máxima ocupação da via.

As regiões definidas no diagrama macroscópio fundamental (que redrelacionam densidade de um fluido com seu fluxo, vide Liebe et al. (2011)), usadas para materiais fluidos, podem ser usadas por analogia para o estudo do fluxo de tráfego de veículos.

A Tabela 1 apresenta a relação entre densidade, fluxo e energia, sendo válida tanto para fluidos materiais quanto para fluxo de tráfego de veículos.

Tabela 1. Relação entre energia, fluxo e densidade.

\begin{tabular}{|c|c|c|}
\hline Densidade & Fluxo & Energia \\
\hline$\rho \leq \rho_{c}$ & fluxo sem retenção & $e_{t}=e_{k}=\frac{1}{2} \rho v^{2}$ \\
\hline$\rho_{c}<\rho \leq \rho_{\max }$ & fluxo congestionado & $\frac{1}{2} \rho v^{2}+\frac{c^{2}}{2 \rho_{c}}\left(\rho-\rho_{c}\right)^{2}$ \\
\hline$\rho=\rho_{\max }$ & fluxo congestionado e saturado & $\frac{c^{2}}{2 \rho_{c}}\left(\rho-\rho_{c}\right)^{2}$ \\
\hline
\end{tabular}

Os valores obtidos pela equação de energia produzem um conjunto de pontos de referência em função dos valores de densidade e de velocidade média de referência das vias em função da energia medida no sistema real. Uma analogia com sistemas elétricos é apresentada por Mayer (2000).

$\mathrm{Na}$ próxima seção, as medidas de energia baseadas em ocupação das vias e velocidade média dos veículos é estudada a partir de um experimento que tem como 
objeto de investigação a influência do tempo de verde dos semáforos sobre os fluxos de energia em um cruzamento.

\subsection{Densidade de movimento}

O lema e o teorema seguintes são usados na formulação do problema proposto, para otimização da energia nas vias.

Lema 1. Seja $\vec{D}(\phi, \gamma)$ um vetor direcional, denominado "densidade de movimento", relacionado com a energia da via, dada pela equação 7 , então existem um $\phi^{*}$ e um $\gamma^{*}$ tais que:

$$
\vec{D}(\phi, \gamma) \leq \vec{D}\left(\phi^{*}, \gamma^{*}\right)
$$

Prova. Como a função 7 é quadrática, fazendo-se: $\frac{\partial \vec{D}(\phi, \gamma)}{\partial \phi}=0$ e $\frac{\partial \vec{D}(\phi, \gamma)}{\partial \gamma}=0$, encontra-se $\left(\phi^{*}, \gamma^{*}\right)$ que minimiza $\vec{D}(\phi, \gamma)$.

Ampliando a avaliação de energia para uma rede de $n$ cruzamentos conectados, estende-se este conceito com o seguinte teorema:

Teorema 2. Seja um conjunto de vetores de densidade de movimento $\vec{D}(\phi, \gamma)_{i} \forall i=1, \ldots, n$ para $n$ cruzamentos. Então existe um valor de densidade de movimento médio, associado à energia da via, $e_{t} \vec{D}(\phi, \gamma)_{\text {médio }}$ tal que

$$
\frac{1}{n}\left|\sum_{i=1}^{n}\right| \vec{D}(\phi, \gamma)_{i}\left|-\vec{D}(\phi, \gamma)_{\text {médio }}\right| \leq \mathcal{C}_{\text {via }}
$$

que minimiza $\mathcal{C}_{\text {via }}$, aumentando a capacidade máxima das vias para um equilíbrio da rede de tráfego.

Prova. Como todas as funções de densidade de movimento nos cruzamentos $\vec{D}(\phi, \gamma)_{i}$ são baseadas na função quadrática 7 restrita a $\phi \geq 0$ e $\gamma \geq 0$, é possível ponderar as equações dos cruzamentos em uma lagrangeana $\mathcal{L}\left(\lambda_{i} \sum_{i} \vec{D}(\phi, \gamma)_{i}\right)$ tal que $\frac{\partial \mathcal{L}(.)}{\partial \rho_{i}}=0$ e $\frac{\partial \mathcal{L}(.)}{\partial v_{i}}=0$.

\section{ESTUDO DE CASO}

Para aplicar a metodologia de modelagem proposta na seção 2.3, são empregados três cruzamentos reais, como mostrado na Figura 3.

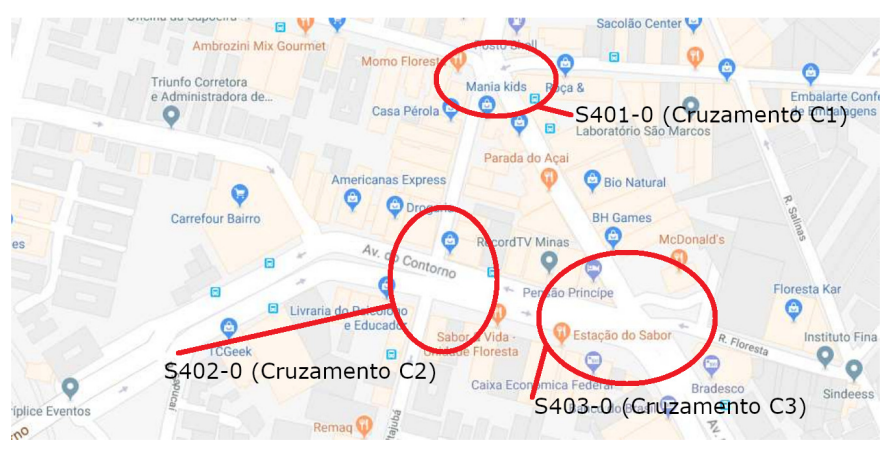

Figura 3. Região do bairro Floresta - BH

\subsection{Adequação do modelo para uma região com três cruzamentos}

A região mostrada na Figura 3 refere-se a um conjunto de interseções que não apresenta a forma regularmente simétrica, exigindo assim especializações para que alguns parâmetros sejam adequados ao modelo proposto.

Os cruzamentos estudados neste trabalho são os encontros das ruas Itajubá, Pouso Alegre, Curvelo e a Avenida do Contorno, todos situados no bairro Floresta, Belo Horizonte, MG. Essa região possui três cruzamentos. O primeiro com sete vias de entrada e cinco de saída, totalizando 12 estados; o segundo com nove vias de entrada e sete vias de saída, totalizando 16 estados e o terceiro com nove vias de entrada e oito vias de saída, totalizando 17 estados.

Ainda sobre a Figura 3, nota-se que o cruzamento estudado tem como entradas a Rua Itajubá, em uma única direção, e a Avenida do Contorno, nas duas direções. O cruzamento possui três acessos monitorados por pontos de medição. Saem da região veículos por três acessos diferentes, sendo um não monitorado (na Rua Itajubá) e dois monitorados.

\subsection{Sinais de entrada e saída da região em estudo}

Para a simulação do modelo, foram feitas repetições de cargas de tráfego no Matlab (para o modelo) e no simulador proprietário $\mathrm{SUMO}^{1}$. Em ambas situações, usou-se como entradas os "tempos de verde". Essas entradas são, neste experimento, sinais pseudo-aleatórios de período variando de três a oitenta segundos para a via principal, podendo ter a repetição de dois a sete ciclos, conforme apresentado na Figura ??. O tempo de amostragem usado foi de 90 segundos (tempo de um ciclo semafórico completo).

O sinal de entrada é aplicado na via principal do cruzamento, como mostra a Figura 4, e o restante do tempo é redistribuído proporcionalmente para as outras fases, nos semáforos das vias secundárias de cada cruzamento.

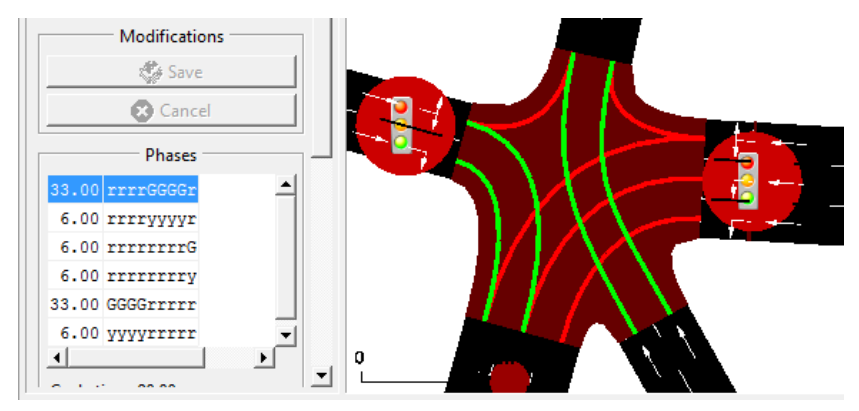

Figura 4. Plano semafórico no simulador SUMO.

\subsection{Descrição do experimento}

A primeira variável de tráfego monitorada foi a ocupação das vias (que representa a porção de tempo durante a qual o detector é ocupado por um veículo, em percentual), como pode ser visto na Figura 6. Algumas vias não sofrem influência direta da ação do sinal, pois derivam de outros cruzamentos.

\footnotetext{
1 SUMO que é uma ferramenta de software de código aberto utilizado para reproduzir condições de qualquer rede de tráfego. É muito utilizado para testar sistemas de controle de tráfego e estratégias de gerenciamento da Engenharia de Trânsito. Foi usada a interface TraCI Manual (Acesso em 02/05/2019) para python para a programação e interface entre o simulador SUMO e a programação e configuração do controle.
} 


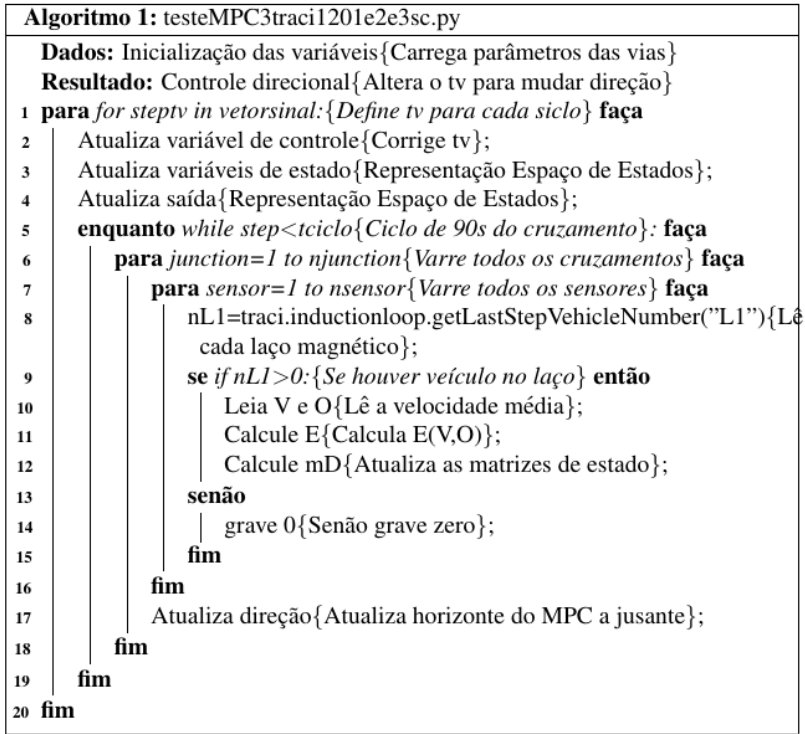

Figura 5. Pseudo-código do experimento

O algoritmo da Figura 5 apresenta um funcionamento integrado do Simulador SUMO que atualiza as variáveis da representação por Espaço de Estados a partir da leitura de todos os sensores (laços magnéticos) de todos os cruzamentos.

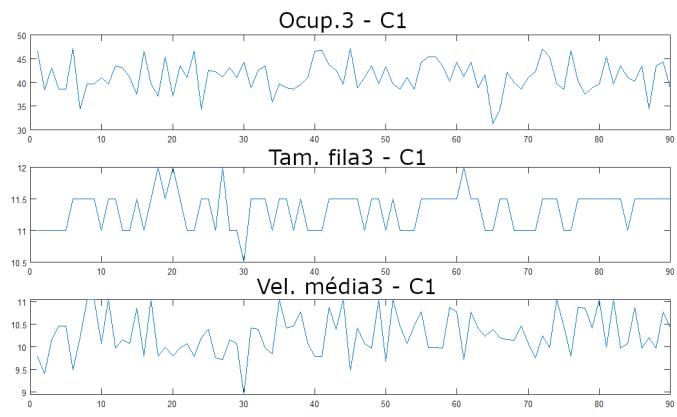

Figura 6. Ocupação das vias, tamanho das filas e velocidade média - Cruzamento 1.

A Figura 6 apresenta as quantidades de carros em cada trecho (ou movimento) que estão prestes a atravessar o cruzamento no início de cada ciclo. O ciclo é definido a partir da soma dos tempos de verde de cada semáforo. Assim, a cada ciclo, todos os semáforos mudam para verde uma vez. Esse tempo é de 90 segundos, para a região em estudo. A velocidade média é registrada em $\mathrm{m} / \mathrm{s}$.

\subsection{Aproximação do modelo}

Uma comparação do modelo apresentado pelas equações 5 e 6 pode ser vista no gráfico da Figura 7, para o tamanho da fila nas vias 2 e 10 do terceiro cruzamento, em relação aos dados reais coletados da BHTRANS (Empresa responsável pelo controle semafórico da cidade de Belo Horizonte).
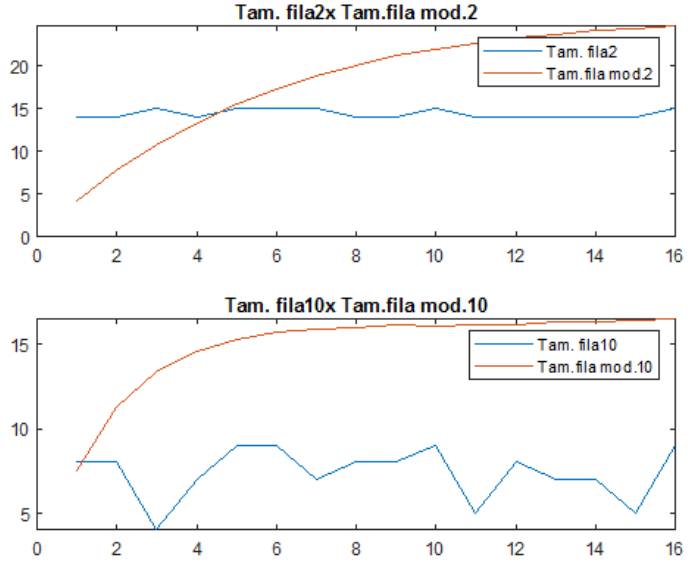

Figura 7. Tamanho da fila no simulador e no modelo

As três variáveis de engenharia de tráfego monitoradas, tamanho da fila, ocupação da via e velocidade média, refletem os estados do modelo descrito na Equação 5.

\section{ANÁLISE}

Para realizar uma análise objetiva da validação do modelo, foi usada a correlação entre a variação das variáveis de trânsito em função da variação da energia, que por sua vez é função do tempo de verde (entrada). Na Figura 8, observou-se um índice de correlação positivo da ocupação da via com o aumento dos tempos de verde, para as vias $3,4,7,8$ e 9 (uma tabela síntese é apresentada na seção de resultados).

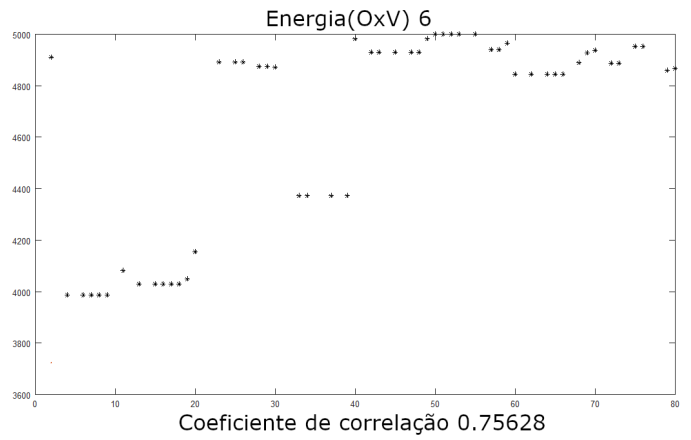

Figura 8. Correlação entre Ocupação x Tempo de verde.

Observou-se também um índice de correlação positivo entre o tamanho da fila de veículos e o aumento dos tempos de verde, para os movimentos 1, 4, 8, 9 e 12. Os movimentos $1,3,8$ e 11 apresentaram um aumento da velocidade média com o incremento do tempo de verde. A energia calculada pela Equação $7, \operatorname{com} c=1$ e $\phi_{c}=1$, indicou um coeficiente de correlação positivo em função do aumento do tempo de verde, para os movimentos $2,6,7$, $8,9,16$ e 18 .

\subsection{Avaliação da variação de energia com as variáveis de tráfego}

Partindo-se da observação anterior de variação de energia nos cruzamentos (energia esta vinculada às variáveis ocu- 
pação e velocidade média), busca-se agora o equilíbrio de energia de uma pequena rede contendo três cruzamentos para uma posterior avaliação da melhoria da ocupação das vias e da velocidade média dos veículos. Foram plotados vetores densidade de energia cuja direção é fornecida por cada segmento de via, como visto na Figura 3 para os três cruzamentos aí presentes. Esta figura apresenta uma sobreposição dos vetores densidade de energia de 100 ciclos de 90 s cujo plano semafórico foi submetido a um sinal de entrada aleatório. O primeiro teste foi feito com um controlador MPC (Model Preditive Control), ver Lopes et al. (2017). Como não há intervenção de um cruzamento em outro, os vetores densidade de energia resultantes dos cruzamentos S401-0 e S402-0 destacam-se em relação ao cruzamento S403-0 que situam-se na região superior, região inferior esquerda e inferior direita do mapa da Figura 3.

Para uma condição de baixa energia, ou seja, com os sinais de tempo de verde variando em torno de $25 \mathrm{~s}$ nos três cruzamentos, os vetores densidade de energia ficam muito pequenos em módulo. Na Figura 9, apresenta-se o resultado de distribuição de energia nos cruzamentos, para 50s de tempos de verde impostos a todos os três cruzamentos.

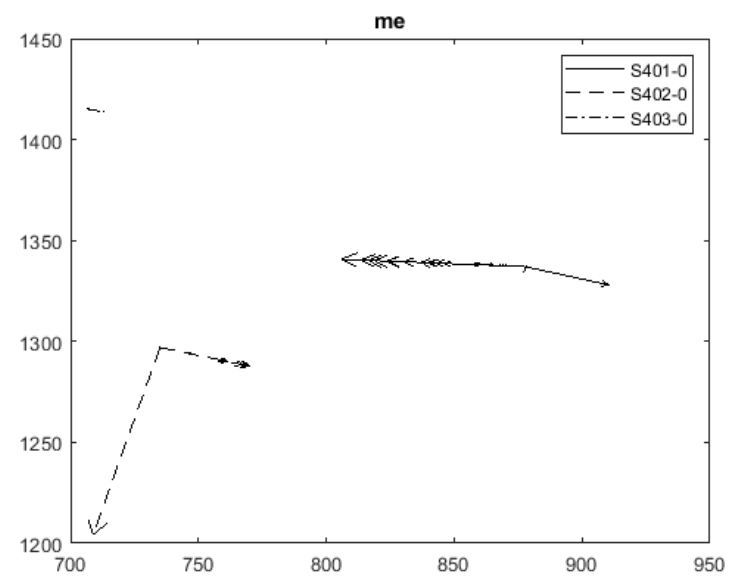

Figura 9. Ponderação de energia nos cruzamentos.

Aplicando-se 75s de tempo de verde em todos os cruzamentos, o equilíbrio da rede também não foi alcançado. Outros três testes foram feitos com a ponderação dos cruzamentos, priorizando um deles em relação aos outros. Foi imposto um tempo de verde de $75 \mathrm{~s}$ para o primeiro cruzamento e de $25 \mathrm{~s}$ para os outros dois. Um mapa de calor (com longitude em x e latitude em y) apresenta uma distribuição gradual de energia em torno dos cruzamentos de interesse, mostrando um vetor resultante da direção para onde aponta o maior fluxo de veículos. Esta informação é utilizada para um controle integrado considerando uma região maior de cruzamentos.

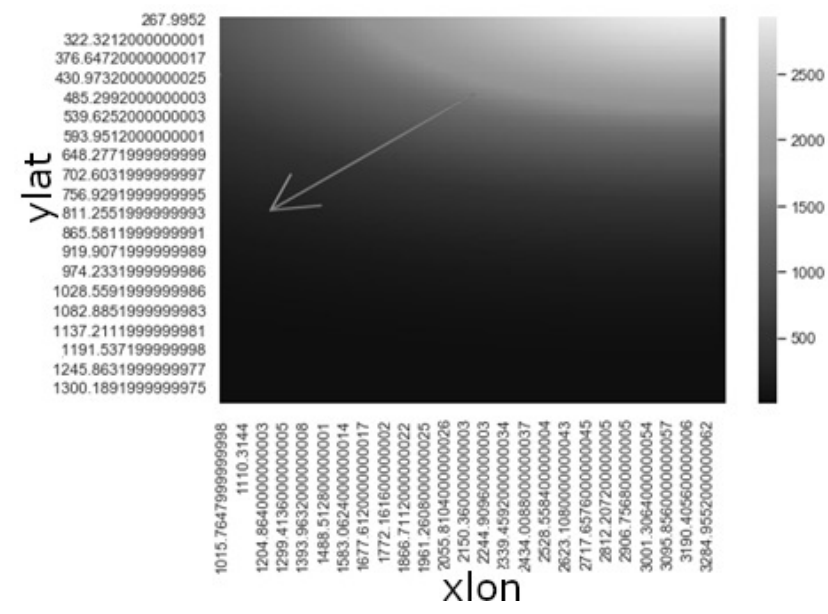

Figura 10. Mapa de calor da ponderação de energia.

Fazendo uma ponderação arbitrária de energia de 50 s para o primeiro e o segundo cruzamentos e de $75 \mathrm{~s}$ para o terceiro, foi possível encontrar uma distribuição mais equilibrada de energia nos três cruzamentos, como se nota na Figura 11.

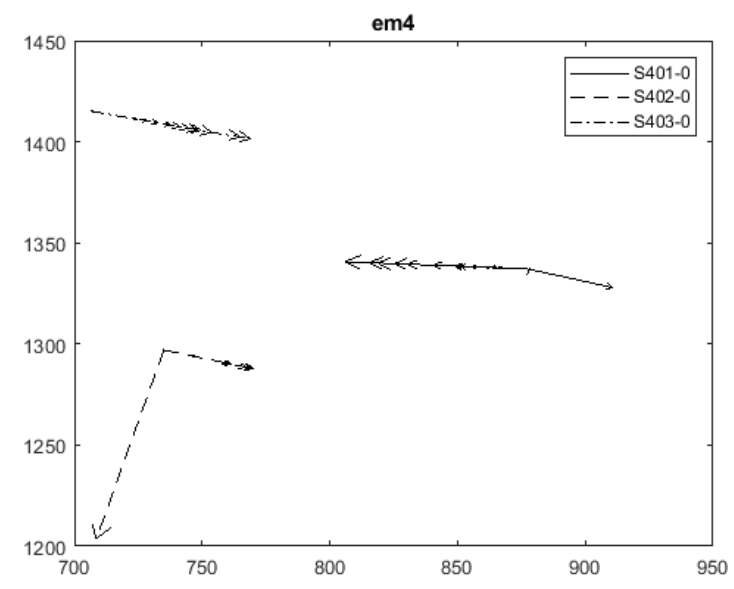

Figura 11. Aproximação do equilíbrio de energia nos três cruzamentos.

A ponderação que levou à melhor distribuição de energia entre os cruzamentos considerou a atribuição de 50s para os tempos de verde dos dois primeiros cruzamentos e de $75 \mathrm{~s}$ para o terceiro. Uma avaliação das variáveis de tráfego será feita em função deste equilíbrio, na próxima seção.

\section{RESULTADOS}

\subsection{Sem equilíbrio de energia}

Comparando os dois últimos testes da seção anterior, quando foi experimentado o valor de $75 \mathrm{~s}$ para o cruzamento S403-0 e de 25 s para os outros dois, havia um claro desequilíbrio de energia e, contabilizando as variáveis de tráfego, percebe-se os valores médios de ocupação das vias em torno de $40 \%$, sendo que cinco movimentos ficam com ocupação quase total. 
O teste com os dois primeiros cruzamentos ajustados para 25 s de tempo de verde e o cruzamento S403-0 para $75 \mathrm{~s}$ levou a um tamanho de fila médio elevado, sobrecarregando uma direção de fluxo. Esta configuração também concentrou a velocidade média em uma via, em detrimento das outras.

A seguir, o resultado do teste citado nesta seção será comparado com o resultado de uma configuração que prioriza o equilíbrio da energia da rede de semáforos.

\subsection{Com equilíbrio de energia}

O equilíbrio de energia entre os cruzamentos foi obtido empiricamente após alguns testes, quando se chegou a uma configuração com 50 s de tempos de verde para os semáforos situados nos cruzamentos S401-0 e S402-0 e 75s de tempo de verde para o semáforo $\mathrm{S} 403-0$. O valor médio da ocupação das vias na Figura 12 aumentou de 1.6592 para 98.3612. O tamanho da fila também aumentou em relação ao ajuste do teste anterior, de 10 para 110 veículos, e o terceiro boxplot mostra um aumento da velocidade média de $0.4717 \mathrm{~m} / \mathrm{s}$ para $1.8013 \mathrm{~m} / \mathrm{s}$.

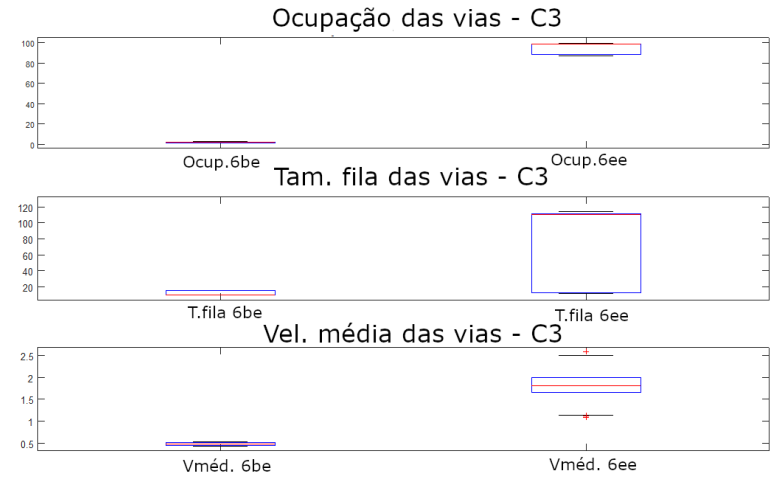

Figura 12. Ocupação da via, tamanho da fila e velocidade média.

Embora se verifique uma redução da velocidade média nos cruzamentos C1 e C3 (Tabela 4), o equilíbrio para toda a rede implica pequenas modificações em várias direções, no sentido de maximizar o desempenho, como uma métrica de avaliação de toda a rede, como será ponderado na próxima subseção.

\subsection{Sintese}

Uma síntese das medidas realizadas nos três cruzamentos, tanto para a ocupação quanto para o tamanho das filas e a velocidade média, é apresentada nas tabelas 2,3 e 4 .

Tabela 2. Taxa de ocupação média dos cruzamentos.

\begin{tabular}{|c|c|c|c|c|}
\hline & C1 & C2 & C3 & Média \\
\hline see & 40,4852 & 56,4249 & 39,9142 & 45,6081 \\
\hline cee & 38,1297 & 55,6893 & 43,2316 & 45,6835 \\
\hline
\end{tabular}

Os três indicadores, avaliados separadamente, não dão uma medida de melhoria sobre o comportamento do sistema de tráfego. O ganho é percebido quando se considera
Tabela 3. Tamanho da fila médio dos cruzamentos.

\begin{tabular}{|c|c|c|c|c|}
\hline & C1 & C2 & C3 & Média \\
\hline see & 14,9241 & 10,4749 & 8,9339 & 11,4443 \\
\hline cee & 16,2764 & 17,1754 & 9,4565 & 14,3028 \\
\hline
\end{tabular}

Tabela 4. Velocidade média dos cruzamentos.

\begin{tabular}{|c|c|c|c|c|}
\hline & C1 & C2 & C3 & Média \\
\hline see & 7,1195 & 7,8871 & 10,4273 & 8,4780 \\
\hline cee & 6,8358 & 8,2492 & 8,2644 & 7,7831 \\
\hline
\end{tabular}

que foi utilizado o mesmo tempo de simulação para os dois cenários e foi obtido um ganho considerável no número de veículos que circulou na rede, de 862 para 1170. Se forem consideradas todas as simulações como corridas acumuladas, foram contados 37.950 veículos antes da intervenção, e 51.497 após a ação de controle.

\section{CONCLUSÃO E TRABALHOS FUTUROS}

Este estudo apresentou uma proposta de modelagem escalável que trata cada cruzamento como um sistema dinâmico representado em espaço de estados, de modo acoplável aos cruzamentos adjacentes e cujas ações de controle podem ser investigadas para possíveis propagações e acoplamentos. Os testes realizados com a busca do equilíbrio de energia trouxe resultados numéricos interessantes, e o uso desta abordagem apontou para uma métrica de redes de cruzamentos com bom potencial para ser estudada em estratégias de controle global de regiões com muitas interseções assimétricas e variantes no tempo.

\section{AGRADECIMENTOS}

Os autores agradecem o Prof. Edward Chung da QUT em Brisbane, Austrália, pelas discussões técnicas, ao CEFETMG pela infraestrutura de pesquisa disponibilizada para a realização deste projeto, à BHTrans pelo fornecimento de dados reais das vias e ao CEFET-MG pelo apoio financeiro para a publicação dos resultados aqui reportados.

\section{REFERENNCIAS}

Borg, D.L. and Scerri, K. (2014). Constrained dynamic control of traffic junctions. 5th International Conference on Ambient Systems, Networks and Technologies(ANT-2014) Procedia Computer Science, 5(32), 293-300.

Borg, D.L. and Scerri, K. (2015). Efficient traffic modelling and dynamic control of an urban region. 4th International Symposium of Transport Simulation ISTS'14, 1-4 June 2014, Corsica, France, 4(6), 224-238.

Daganzo, C.F. (1997). Fundamentals of transportation and traffic operations. Pergamon.

David, S., Eisele, B., and Lomax, T. (2012). Urban mobility report. Texas transportation institute's annual urban mobility report.

Halaoui, H.F. (2010). Intelligent traffic system: Road networks with weighted time graphs. International Journal for Infonomics, 3(4).

Homolova, J. and Nagy, I. (2005). Traffic model of a microregion. 16th Triennial World Congress, Prague, Czech Republic. 
Liebe, C., Kuhne, R., and Wang, H. (2011). Traffic flow prospectives: From fundamental diagram to energy balance. TRANSPORTATION RESEARCH CIRCULAR, $\mathrm{E}(\mathrm{C} 149), 63-72$.

Lopes, R.C., Miranda, M.F., and Almeida, P.E.M. (2017). Ltv-mpc: Uma nova abordagem para a temporização semafórica urbana a partir de controle preditivo. SBAI - XIII Simpósio Brasileiro de Automação Inteligente, 2295-2300.

Manual, S. (Acesso em 02/05/2019). Traci/interfacing traci from python. https://sumo.dlr.de/.

Mayer, D. (2000). Variation principles and theory of electrical circuit. Casopis EE, 6(5), 4-7.

Morrison, P.J. (1998). Hamiltonian description of the ideal fluid. Reviews of Modern Physics, 70(2), 467-521.

Nakrachi, A., Hayat, S., and Popescu, D. (2012). An energy concept for macroscopic traffic flow modelling. Eur. Transp. Res. Rev., 0(4), 57-66.

Pecherkova, P., Dunik, J., and Flidr, M. (2008). Modelling and simultaneous estimation of state and parameters of traffic system. Robotics, Automation and Control, 319336.

Pedersen, N.J. (2011). 75 Years of the Fundamental Diagram for Traffic Flow Theory. Massachussets, Traffic Flow Theory and Characteristics Committee.

\section{Apêndice A. REPRESENTAÇÃO DE UM SISTEMA DE TRÁFEGO POR ESPAÇO DE ESTADOS}

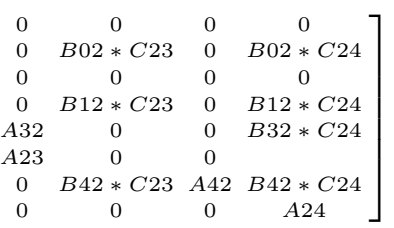

$$
B 1=\left[\begin{array}{cccc}
B 20 & 0 & 0 & 0 \\
0 & B 02 * D 21 & B 02 * D 23 & B 02 * D 24 \\
0 & B 21 & 0 & 0 \\
B 12 * D 20 & 0 & B 12 * D 23 & B 12 * D 24 \\
B 32 * D 20 & B 32 * D 21 & 0 & B 32 * D 24 \\
0 & 0 & B 23 & 0 \\
B 42 * D 20 & B 42 * D 21 & B 42 * D 23 & 0 \\
0 & 0 & 0 & B 24
\end{array}\right]
$$

$C 1=\left[\begin{array}{cccccccc}0 & C 02 & D 02 * C 21 & 0 & 0 & D 02 * C 23 & 0 & D 02 * C 24 \\ D 12 * C 20 & 0 & 0 & C 12 & 0 & D 12 * C 23 & 0 & D 12 * C 24 \\ D 32 * C 20 & 0 & D 32 * C 21 & 0 & C 32 & D 32 * C 23 & 0 & D 32 * C 24 \\ D 42 * C 20 & 0 & D 42 * C 21 & 0 & 0 & D 42 * C 23 & C 42 & 0\end{array}\right]$

$$
D 1=\left[\begin{array}{cccc}
0 & D 02 * D 21 & D 02 * D 23 & D 02 * D 24 \\
D 12 * C 20 & 0 & D 12 * D 23 & D 12 * D 24 \\
D 32 * D 20 & D 32 * D 21 & 0 & D 32 * D 24 \\
D 42 * D 20 & D 42 * D 221 & D 42 * D 23 & 0
\end{array}\right]
$$

Este sistema possui vinte e quatro estados, considerando, por exemplo que a célula $A 1(1,1)$ da matriz $A 1$ é a matriz $3 \times 3$ A20 equivalente à matriz $A$ da equação 5 com três estados. Como o cruzamento completo possui quatro vias (links, ou movimentos) que levam para dentro do cruzamento e mais quatro movimentos para fora do cruzamento, totalizando oito segmentos, totalizam-se os vinte e quatro estados. A matriz $B 1$ possui quatro colunas que significam quatro acessos para dentro da interseção e a matriz $C 1$ tem quatro linhas que representam as quatro saídas do cruzamento. A matriz $D 1$ geralmente é composta por zeros, significando que não há uma transmissão direta das entradas para as saídas.

\section{Apêndice B. DADOS MEDIDOS NAS VIAS DOS CRUZAMENTOS}

Nas tabelas 1, 2 e 3 foram registradas as medidas de ocupação das vias, tamanho da fila e velocidade média para o cruzamento 3 antes e depois do ajuste para equilíbrio da energia da rede de tráfego.

Tabela B.1. Taxa de ocupação - Cruzamento 3.

\begin{tabular}{|c|c|c|c|c|c|c|c|}
\hline see & 25.4999 & 28.9157 & 34.6635 & 22.2026 & 1.0324 & 95.3138 & 92.3606 \\
\hline cee & 19.2969 & 29.1885 & 32.1592 & 6.0039 & 3.4815 & 93.8058 & 91.2465 \\
\hline
\end{tabular}

A ocupação diminuiu em quase todas as vias, significando que cada veículo fica um tempo menor sobre o sensor magnético.

Tabela B.2. Tamanho da fila - Cruzamento 3.

\begin{tabular}{|c|c|c|c|c|c|c|c|}
\hline see & 0.77778 & 7.6333 & 4.0611 & 0.47778 & 0.022222 & 74 & 41.3333 \\
\hline cee & 0.65556 & 7.6778 & 4 & 0.12222 & 0.077778 & 70.3667 & 37.0667 \\
\hline
\end{tabular}

Algumas vias aumentaram o tamanho médio das filas e outras diminuiram, quando foram ajustados os tempos de verde para a busca do equilíbrio de energia na rede.

Tabela B.3. Velocidade média - Cruzamento 3.

\begin{tabular}{|c|c|c|c|c|c|c|c|}
\hline see & 10.6329 & 10.5436 & 10.2753 & 5.4295 & 0.23917 & 1.8114 & 5.0131 \\
\hline cee & 8.8652 & 10.625 & 10.1683 & 1.3719 & 0.76773 & 1.7967 & 4.6841 \\
\hline
\end{tabular}

O mesmo efeito ocorreu com a velocidade média, aumentado em algumas vias e reduzindo em outras. 